University of Rhode Island

DigitalCommons@URI

Open Access Master's Theses

2012

\title{
A PROSPECTIVE EXAMINATION OF POSTTRAUMATIC STRESS AND ALCOHOL USE DISORDERS AMONG RETURNING VETERANS
}

Matthew D. Pierce

University of Rhode Island, mpie0351@my.uri.edu

Follow this and additional works at: https://digitalcommons.uri.edu/theses

\section{Recommended Citation}

Pierce, Matthew D., "A PROSPECTIVE EXAMINATION OF POSTTRAUMATIC STRESS AND ALCOHOL USE DISORDERS AMONG RETURNING VETERANS" (2012). Open Access Master's Theses. Paper 109.

https://digitalcommons.uri.edu/theses/109

This Thesis is brought to you for free and open access by DigitalCommons@URI. It has been accepted for inclusion in Open Access Master's Theses by an authorized administrator of DigitalCommons@URI. For more information, please contact digitalcommons-group@uri.edu. 
A PROSPECTIVE EXAMINATION OF POSTTRAUMATIC STRESS AND ALCOHOL USE DISORDERS AMONG RETURNING VETERANS BY MATTHEW D. PIERCE

A THESIS SUBMITTED IN PARTIAL FULFILLMENT OF THE REQUIREMENTS FOR THE DEGREE OF

MASTER OF ARTS

IN PSYCHOLOGY

UNIVERSITY OF RHODE ISLAND 
MASTER OF ARTS IN PSYCHOLOGY THESIS

OF

MATTHEW D PIERCE

APPROVED:

Thesis Committee:

Major Professor Mark Wood

Paul Florin

Sandy Jean Hicks

Nasser H. Zawia

DEAN OF THE GRADUATE SCHOOL

UNIVERSITY OF RHODE ISLAND

2012 


\begin{abstract}
The traumatic effects of combat on psychological functioning have long been recognized. Operation Iraqi Freedom and Operation Enduring Freedom have amplified the need for a better understanding of the association between posttraumatic stress disorder (PTSD) and alcohol use disorders (AUD). We prospectively examined PTSD and AUD symptoms and investigated relations between combat exposure (CE) and relationship status (RS) on these outcomes. We hypothesized that CE would have a main effect on both PTSD and AUD symptoms while RS would have a main effect on AUD symptoms but not PTSD. We also examined CE X RS interactions as well as hypothesized reciprocal prospective PTSD and AUD associations. Participants ( $N=238,92 \%$ men) were National Guard and Reserve Soldiers returning from deployment, recruited as part of a larger study. Twelve month data were available for $66.8 \%$ of the initial sample with no evidence of differential attrition according to baseline AUD, PTSD or CE. We examined both PTSD and AUD symptoms for months one, six and 12 post-deployment, derived from initial and follow-up clinical interviews. Assessments consisted of the Clinician Administered PTSD Scale (CAPS) and the Longitudinal Interval Follow-up Evaluation (LIFE). We conducted separate (RS X CE X Time) mixed-design ANOVAs on our primary measures of interest (PTSD \& AUD). RS was coded as single/divorced vs. married/cohabitating and CE was categorized according to "minimal", "moderate" and "severe" levels. CE was significantly associated with PTSD but no other main or interaction effects emerged. To examine hypothesized reciprocal associations between PTSD and AUD we
\end{abstract}


conducted cross-lagged panel path models with RS, CE and the RS X CE interaction as manifest exogenous variables. In separate models we examined alcohol abuse or dependence vs. dependence alone. CE consistently predicted Time 1 PTSD symptoms and RS predicted Time 1 alcohol dependence, but in contrast to our hypotheses no other main or interaction effects were observed including anticipated reciprocal relations between PTSD and AUD. In large part, this is due to strong (i.e., > .8) autoregressive effects across the three time points. Findings support hypothesized effects of CE on PTSD symptoms but the lack of observed hypothesized associations elsewhere suggests the need for longer measurement intervals in prospective research, as well as larger and more diverse samples. Supported by Department of Defense grant (W81XWH-06-1-0573). 


\section{ACKNOWLEDGMENTS}

I would like to recognize the support and guidance of my thesis committee for their insight and flexibility regarding this process. Specifically, I would like to thank Dr. Mark Wood for his patience, compassion and mentorship in developing my skills as a young researcher. Additionally, I need to recognize the hard work and dedication of Dr. M. Tracie Shea and her staff from Brown University and the Providence VA Medical Center. Their previous studies and collection of the data used in this study were of the utmost importance in allowing me the opportunity to contribute my efforts

for this thesis. Lastly, I would like to acknowledge the military service members who participated in this study for their contributions towards a better understanding of how to best treat and prevent these types of mental health concerns in the future. 


\section{TABLE OF CONTENTS}

$\begin{array}{lll}\text { ABSTRACT } & \text { ii }\end{array}$

ACKNOWLEDGMENTS

TABLE OF CONTENTS r v

LIST OF TABLES vi vi

LIST OF FIGURES vii

CHAPTER 1

INTRODUCTION 1

CHAPTER 2

REVIEW OF LITERATURE 2

$\begin{array}{ll}\text { CHAPTER } 3 & 13\end{array}$

$\begin{array}{ll}\text { METHODOLOGY } & 13\end{array}$

$\begin{array}{ll}\text { CHAPTER } 4 & 20\end{array}$

$\begin{array}{ll}\text { FINDINGS } & 20\end{array}$

$\begin{array}{ll}\text { CHAPTER 5 } & 26\end{array}$

$\begin{array}{ll}\text { CONCLUSION } & 26\end{array}$

$\begin{array}{ll}\text { BIBLIOGRAPHY } & 37\end{array}$ 


\section{LIST OF TABLES}

TABLE

PAGE

Table 1. Descriptive statistics for AUD, PTSD and Combat Exposure 


\section{LIST OF FIGURES}

FIGURE

PAGE

Figure 1. CE effect on PTSD 35

Figure 2. Time effect on PTSD. 35

Figure 3. Cross-lagged Panel Path Analysis Model 36 


\section{CHAPTER 1}

\section{INTRODUCTION}

The traumatic effects of combat on psychological functioning have long been recognized. However, Operation Iraqi Freedom (OIF) and Operation Enduring Freedom $(\mathrm{OEF})$ have amplified the need for a better understanding of the association between posttraumatic stress disorder (PTSD) and other serious mental health concerns that often co-occur with PTSD, such as alcohol use disorders. Current research has been largely limited to cross-sectional or brief self-report surveys that are not well suited to capture the dynamic interplay of alcohol use and PTSD among returning veterans over time. Accordingly, the major goal of the current study is to extend previous research by longitudinal examination of the course of PTSD and to investigate the association of PTSD symptoms with alcohol use disorder symptomatology. The following will review PTSD and alcohol use disorders as well as their unique relationship to military populations as discussed in recent studies. 


\section{CHAPTER 2}

\section{REVIEW OF LITERATURE}

\section{PTSD\& AUDs in Veterans:}

The existing literature on returning veterans shows that prevalence rates for PTSD and alcohol use disorders are high and escalating, indicating a dire need for interventions for these disorders. Researchers have found that rates of PTSD are more than twice as high in military veterans compared to civilians (Hoge, 2004; Kulka, 1990; National Comorbidity Survey, 2005). Likewise, alcohol use and alcohol use disorders are significantly higher than their civilian counterparts regardless of gender or age (Bray, Marsden, \& Peterson, 1991; Bray et al., 2010; Fear et al., 2007; Stahre, Brewer, Fonseca \& Naimi, 2009).

\section{PTSD: Definitions and Prevalence}

According to the Diagnostic and Statistical Manual of Mental Disorders (DSM-IV-TR), PTSD is an anxiety disorder that involves an exposure to a traumatic event that involves actual or threatened death or serious injury. The characteristic symptoms resulting from the exposure to extreme trauma include persistent reexperiencing of the traumatic event, sustained avoidance of stimuli associated with the trauma and numbing of general responsiveness and prolonged symptoms of increased arousal. The full symptoms must be present for over one month and the symptoms must cause clinically significant distress or impairment in social, occupational or other important areas of functioning (American Psychiatric Association, 2000). 
Recognition of the severe impact of combat related stress long preceded its formal recognition as a clinical disorder (Shephard, 2001). Combat stress reaction, historically known as shell shock or battle fatigue, is a term used to categorize a range of behaviors resulting from the stress of battle which decrease the combatant's fighting efficiency. The most common symptoms are fatigue, slower reaction times, indecision, disconnection from one's surroundings and an inability to prioritize. Combat stress reaction is generally short term, which distinguishes it from acute stress disorder or the more pervasive and dysfunctional PTSD. The modern understanding of PTSD dates from the 1970's largely as a result of the problems experienced by U.S. military veterans returning from Vietnam. The condition was formally recognized as a disorder when it was included in the third edition of the DSM in 1980 (America Psychiatric Association, 1980).

Although PTSD can occur following any traumatic event the prevalence rates for civilians is markedly lower than that of military personnel. In studies comparing civilians to military veterans across different wars from Vietnam to OEF/OIF findings show that veterans are two to three more times likely to develop PTSD than their civilian counterparts (Kulka, 1990; National Comorbidity Survey, 2005). The most comprehensive study of PTSD rates for current conflicts estimated the risk at $11 \%$ for service members participating in OEF and $18 \%$ for service members deployed to OIF (Hoge et al, 2004). Although this high rate is comparable with past conflicts it may still be under reported. Researchers believe that prevalence rates may be higher because the use of self-report measures and the timing of military assessments may not accurately reflect actual prevalence of PTSD in returning veterans (Warner et al, 
2011). To assess the timing of assessment issue, Bliese, Wright, Adler, Thomas and Hoge, (2007) identified factors that would explain lower rates of PTSD symptoms upon immediate reintegration as compared to symptoms at 120 days post-deployment. These factors include: a sense of relief about having returned home safely, reluctance to report symptoms out of fear that medical treatment would interfere with taking time off, and that certain symptoms might take more time to develop into problems based on changes in routine and adjustments to interpersonal relationships (Bliese et al., 2007). Additionally, it has also been suggested that potential stigma towards mental health services is preventing those with PTSD from reporting symptoms and seeking the help they need (Hoge et al., 2004; Kim, Thomas, Wilk, Castro \& Hoge, 2010). These factors further complicate the understanding of how and when PTSD symptoms develop following deployments, which is one of the important questions the current research will address.

According to the Deployment Health Clinical Center, the military currently assesses for mental health following deployments by using the Post-Deployment Health Assessment (PDHA) immediately upon return and the Post-Deployment Health Re-Assessment (PDHRA) three to six months later (http://www.pdhealth.mil/dcs/pdhra.asp). These screenings are required on-line selfreport measures which are then reviewed and discussed with a healthcare provider to allow appropriate referrals for services. The completed form then becomes part of the service member's individual permanent medical record. Research using this measure has shown that mental health concerns including PTSD can be identified early but later assessments show higher prevalence rates and that the measure is only as reliable 
as the service member's willingness to disclose information honestly (Allison-Aipa, Ritter, Sikes \& Ball, 2010; Milliken, Auchterlonie, \& Hoge, 2007).

Military veterans of OEF and OIF are subject to a multitude of different factors than previous veterans, which can make predicting PTSD even more difficult. Notably, the length of military involvement and an all volunteer military has produced longer and more frequent deployments, which has increased the chances for combat exposures. Research has shown that besides being wounded or injured, there is a strong correlation between combat experiences, such as being shot at, handling dead bodies, knowing someone who was killed, or killing enemy combatants and the prevalence of PTSD (Hoge et al., 2004; Litz, 2007).

The prolonged and dual combat zones have also increased the use of Reserve Component and National Guard units to support both wars. This is of particular interest because this population comprises approximately $40 \%$ of OEF/OIF troops and has been shown to be at higher risk of PTSD than active duty personnel following deployment (Allison-Aipa et al., 2010; Kim et al., 2010; Milliken et al., 2007). Some potential reasons for differences from active duty forces are that reservists are more likely to experience unexpected disruption to families, careers and resulting financial pressures may have been contributory to problems at home. Reservists have reported higher exposure to traumatic experiences, lower unit cohesion, more problems adjusting to homecoming and lower marital satisfaction (Browne et al., 2007).

As veterans transition from either a war zone to being at home again or from active military service to becoming a civilian again, there are many individual factors that can influence the ease of this transformation. It is because of these factors that 
some veterans experience adjustment problems at different times and often may end up suffering from a myriad of mental health disorders. PTSD does not always develop immediately following a particular trauma; in fact many cases develop as delayed onset PTSD, which is defined as meeting criteria more than six months after a traumatic event (American Psychiatric Association, 2000). Although the extant literature on this question is sparse, recent studies report delayed onset PTSD accounts for $15 \%$ of civilian PTSD cases and $38 \%$ of military PTSD cases (Andrews, Brewin, Stewart, Philpott \& Hejdenberg (2009). Moreover, the delayed onset group was significantly more likely to have episodes of major depression and alcohol abuse at some period within service prior to their PTSD onset. This underscores the need for prospective research that can investigate reciprocal associations between problematic alcohol use and PTSD. Delayed onset PTSD is characteristic of individuals who develop general stress responses in a cumulative fashion rather than characteristic of those who are overwhelmed by a single traumatic experience (Andrews et al., 2009). This complex type of PTSD could explain why symptoms develop later rather than at initial redeployment but also provides impetus for the question of whether alcohol use is a contributing factor for the development of PTSD or another way of coping with PTSD symptoms afterwards.

Although the mechanisms by which trauma exposure leads to alcohol use and/or PTSD in veteran populations may differ and remain unclear, the prevalence of dual diagnosis alcohol use disorders (AUD) and PTSD among veteran populations has been increasing over time (Seal, Bertenthal, Miner, Sen, \& Marmar, 2007). Additionally, individuals with comorbid AUD and PTSD are more likely to have 
attempted suicide and to report lower quality of life (Leeies, Pagura, Sareen, \& Bolton, 2010). Further, veterans with PTSD who also met criteria for AUD are more likely to display persistent and declining effects on social functioning and have lower rates of employment (Fontana \& Rosenheck, 2010). Given the high rates of comorbidity between PTSD and AUD, next we briefly review AUD with emphasis on military populations.

\section{Alcohol Use and Alcohol Use Disorders}

Alcohol use is typically measured by assessing quantity and frequency of alcohol consumption as well as identifying heavy episodic or "binge" drinking. Heavy episodic drinking is typically defined as five or more drinks for men or four or more drinks for women on any single occasion (National Institute of Alcohol Abuse and Alcoholism, 2004).

The DSM-IV-TR categorizes alcohol use disorders as either alcohol abuse or dependence. Alcohol abuse is defined as an unhealthy pattern of alcohol use exhibited by recurrent and significant adverse consequences related to the repeated use. Criteria include persistent alcohol use resulting in difficulty at work, school or home, use in situations which are physically hazardous, produce legal problems, and continued use despite having persistent or recurrent interpersonal problems caused or exacerbated by the alcohol use (American Psychiatric Association, 2000).

Alcohol dependence is defined as an unhealthy pattern of use leading to clinically significant impairment or distress as exhibited by three or more of the following criteria. The criteria are tolerance, withdrawal, drinking more or for longer than intended, a desire to or unsuccessful efforts to reduce drinking, a large amount of 
time used in obtaining, using or recovering from alcohol, loss of interest in important activities, and persistent alcohol use despite knowledge of having a problem (American Psychiatric Association, 2000).

According to the NIAAA's 2001-2002 National Epidemiological Survey on Alcohol and Related Conditions (NESARC: $N=43,093$ ), the civilian prevalence rates for alcohol abuse was 4.65 percent and alcohol dependence was 3.81 percent. This study found that abuse and dependence were more common among males and among younger respondents (Grant et al., 2006).

Several studies of health related behaviors among military personnel have shown that active duty military personnel (ADMP) have substantially higher rates of heavy drinking than their civilian counterparts regardless of gender or age (Bray, Marsden, \& Peterson, 1991; Bray et al., 2010; Fear et al., 2007; Stahre, Brewer, Fonseca \& Naimi, 2009). Compared to non-heavy drinkers, heavy drinkers, as defined by more than two drinks a day for men and more than one drink for women, were more likely to report alcohol-related harms, including job performance problems; alcohol impaired driving and criminal justice problems. The high levels of binge drinking among ADMP, particularly those aged $\leq 26$ years, increases the likelihood of alcohol-related harms and alcohol-use disorders following military service (Stahre et al., 2009). Recent studies within the Department of Veterans Affairs (VA) have found that hazardous alcohol use, as measured by the Alcohol Use Disorder Identification Test (AUDIT), is prevalent among OEF/OIF veterans seeking VA health care and that hazardous drinking is significantly correlated with PTSD symptoms (Calhoun, Elter, 
Jones, Kudler, Straits-Troster, 2008; Hawkins, Lapham, Kivlahan, Bradley, 2010;

McDevitt-Murphy et al., 2010).

Just as perceived combat experience was related to predicting PTSD, research has shown that higher levels of reported combat exposure is also associated with higher levels of alcohol use among returning veterans (Jacobson et al., 2008; Thomas et al., 2010; Wilk et al, 2010).

Although the mechanisms by which trauma exposure lead to alcohol use and/or PTSD in veteran populations may differ and remain unclear, the prevalence of dual diagnosis AUDs and PTSD among veteran populations has been increasing over time (Seal, Bertenthal, Miner, Sen, \& Marmar, 2007). Additionally, individuals with comorbid AUD and PTSD are more likely to have attempted suicide and to report lower quality of life (Leeies, Pagura, Sareen, \& Bolton, 2010). Further, veterans with PTSD who also met criteria for AUD are more likely to display persistent and declining effects on social functioning and have lower rates of employment (Fontana \& Rosenheck, 2010).

One explanation regarding increased prevalence of PTSD is the increase in combat exposure from multiple deployments and today's non-linear battlefield. Research has shown that besides being wounded or injured, there is a strong correlation between combat experiences, such as being shot at, handling dead bodies, knowing someone who was killed, or killing enemy combatants and the prevalence of PTSD (Hoge et al., 2004; Litz, 2007). Just as perceived combat experience was related to predicting PTSD, research has shown that higher levels of reported combat 
exposure is also associated with higher levels of alcohol use among returning veterans (Jacobson et al., 2008; Thomas et al., 2010; Wilk et al, 2010).

\section{Limitations of Previous Research}

A majority of existing studies on PTSD among veterans have focused on risk factors and prevalence rates but have not examined specific behaviors that co-occur such as alcohol use or, particularly, alcohol use disorders (Bliese et al., 2007; Hoge et al., 2004; Kim et al., 2010; Milliken et al., 2010). Other studies have focused on military populations and alcohol use but not specific to PTSD comorbidity (Calhoun et al., 2008; Hawkings et al., 2010; McDevitt-Murphy et al., 2010). Studies jointly examining, PTSD and alcohol use/disorders observed high levels of comorbidity and strong associations but have not examined reciprocal relations over time using more comprehensive measures such as diagnostic interviews (Allison-Aipa et al., 2010; Jacobson et al., 2010; Thomas et al., 2010).

To address these shortcomings in the literature, the current research will examine levels of PTSD and AUD symptomatology over time and investigate relations between combat exposure and relationship status on these outcomes. We will also prospectively examine reciprocal relations between PTSD and AUD symptoms using both self-report and clinician administered assessments on a relevant population of recently redeployed National Guard and Reserves troops from OEF/OIF. This study will also investigate the potential influence of combat exposure on the reciprocal interplay of PTSD and AUD symptoms for 12 months subsequent to the end of redeployment.

\section{Research Aims \& Hypotheses}


Based on the brief literature review above, there are several unanswered questions that the present study seeks to address. With a limited amount of prospective research at this time this study will provide an understanding regarding issues of timing and assessment, as well as dynamic relations between AUD and PTSD over time and the impact of combat level exposure on both AUD and PTSD immediately subsequent to deployment to 12 months post-deployment.

\section{Aim 1: Prospective Examination of PTSD and AUD}

In separate mixed-design Analyses of Variance (ANOVAs) we examined mean levels of PTSD symptomatology and AUD diagnoses over time in relation to combat exposure and relationship status. Prospective examination of both AUD diagnoses and, particularly, PTSD symptomatology can provide important information on whether symptoms increase, decrease, or persist over time and the effects of combat exposure and relationship status on any observed variation. We hypothesized a main effect for combat exposure, such that higher levels of exposure would predict greater PSTD symptoms and AUD diagnoses (Hypotheses $1 \mathrm{a}-1 \mathrm{~b}$ ). We hypothesized a main effect of relationship status on AUD diagnoses, such that individuals in relationships would be less likely to meet AUD criteria, but not for PTSD symptomatology (Hypothesis 2). We further hypothesized that relationship status would mitigate the effects of combat exposure on PTSD symptomatology, such that PTSD symptoms would be reduced among individuals who are married or cohabitating compared to individuals that are single, divorced or widowed. We also explored the same possible protective effect for relationship status for the prediction of AUD diagnoses from combat exposure (Hypothesis 3). 


\section{Aim 2: Prospective Examination of Associations between PTSD and AUD}

\section{Symptomatology}

Using structural equation modeling in a cross-lagged panel design, we prospectively examined PTSD and AUD symptom relations over a 12 month period. We hypothesized that there would be reciprocal prospective associations between PTSD and AUD (Hypothesis 4) after controlling for both cross-sectional and autoregressive (e.g., AUD T1 to AUD T2) effects.

\section{Aim 3: Influence of Combat Exposure on AUDs and PTSD Symptomatology}

As previously noted, a number of studies have shown that combat exposure is associated with higher levels of alcohol use and prevalence of PTSD. However, less is known about the severity and timing of these associations. Accordingly, we also examined whether combat exposure prospectively predicts AUD and PTSD symptoms at six and 12 month follow-up intervals with the hypotheses that combat exposure will be positively associated with changes in both PTSD and AUD symptoms over time (Hypothesis 5). 


\section{CHAPTER 3}

\section{METHODOLOGY}

\section{Study Background and Procedure}

Data for the current study are drawn from a larger study of OEF/OIF National Guard and Reserve veterans (Shea, Vujanovic, Mansfield, Sevin \& Liu, 2010). Participants were recruited from Rhode Island National Guard and Reserve units following OEF/OIF deployments. All returning unit personnel were eligible to participate unless psychosis or other conditions characterized by cognitive impairment would prevent a valid interview were present (none met this exclusion criteria). The study received approval from the human subjects review boards at Brown University, the Department of Veterans Affairs, and the Department of Defense.

As part of the larger study (Shea et al., 2010), service personnel who had recently completed deployments were recruited and assessed using an extensive battery of items to assess background and demographic factors, as well as diagnostic assessment of PTSD, AUD, and related risk and protective factors. Participants completed a combination of self-report questionnaires and diagnostic interviews across four different time points. Trained interviewers with at least a Master's level degree or a minimum two years supervised experience in conducting clinical interviews were responsible for the administration of the data collection process. Informed consent and participant interviews took place at either the Providence VA Medical Center or Brown University offices. Initial assessments (Time 1) were 
administered within six months of redeployment with follow-up assessments taken six months after initial assessment (Time 2), 12 months after initial assessment (Time 3) and 24 months after initial assessment (Time 4). Because of high rates of attrition by Time 4, the current study will utilize data from the first three time points to examine the interrelations of AUD and PTSD symptoms from early redeployment to at least twelve months post-deployment.

\section{Sample}

The researchers were able to present this study to about $67 \%$ of the military personnel returning from the units approached with $66 \%$ of those hearing about the study agreeing to be contacted and $70 \%$ of those personnel contacted agreeing to participate (Shea et al., 2010). Thus, there were 238 participants at the initial assessment which included 220 males (92\%) and 18 women (8\%), with an average age of $33.5(\mathrm{SD}=9.5)$. In terms of race/ethnicity, $209(87.8 \%)$ participants identified as Caucasian; 17 (7.1\%) identified as African American; and $12(5.1 \%)$ identified as other minority groups. Thirty-four participants (14.3\%) identified as Hispanic/Latino. Ninety-three participants (39.1\%) were single, 108 (45.4\%) were married or living with a partner, $36(15.1 \%)$ were divorced or separated, and $1(0.4 \%)$ was widowed. Most (51.7\%) reported some post-high school education, 29 (12.2\%) reported graduating from college, and $12(5.0 \%)$ reported holding a post-graduate degree. As noted earlier, there was some level of attrition throughout the course of the study with $44(18 \%)$ participants dropping out between Time 1 and Time 2 and 32 (13\%) participants dropping out between Time 2 and Time 3 . Therefore, a cumulative 
attrition rate of approximately 32\% occurred between Time 1 (238) and Time 3 (162) which was investigated for any possible systematic or symptomatic explanations.

\section{Measures}

Participant demographic information such as gender, age, ethnicity, education and employment information was gathered from a self-report participant information form at the initial assessment. This form also captured contact information of the participant as well as additional family contact information in case of difficulty contacting subjects with follow-up assessments.

PTSD was assessed at all time points using the Clinician Administered PTSD Scale for DSM-IV (CAPS) and the Longitudinal Interval Follow-up Evaluation (LIFE). The CAPS assessment measures both current and lifetime prevalence of PTSD based on thorough evaluation of symptom frequency and intensity. The LIFE consists of several sections which include lifetime and current diagnoses as well as changes in symptom levels across time. PTSD and AUD were evaluated at the symptom level and scored continuously for a more detailed comparison.

AUDs were also assessed using the LIFE as explained above. The criteria for both alcohol abuse and dependence are equivalent to the Diagnostic Statistical Manual, $4^{\text {th }}$ Edition, Text Revision (American Psychiatric Association, 2000).

Individual combat exposure (CE) was assessed by using the Hoge Combat Experiences Survey (HOGE). This self-report measure was designed to assess exposure to combat and other war zone experiences in Iraq and Afghanistan (Hoge et al., 2004).

\section{Measurement Diagnostic Information}




\section{PTSD}

PTSD was assessed at all time points using the Clinician Administered PTSD Scale for DSM-IV (CAPS) and the Longitudinal Interval Follow-up Evaluation (LIFE). The CAPS measures both current and lifetime prevalence of PTSD. The LIFE consists of several sections which include lifetime and current diagnoses as well as changes in symptom levels across time.

The CAPS is a 30-item structured interview designed to assess the 17 symptoms of PTSD, 8 hypothesized associated features, and global ratings of subjective distress and psychosocial functioning. The hypothesized features include questions regarding guilt, reduction in awareness, and depersonalization not specifically covered by the 17 DSM related symptoms. The scale produces a dichotomous diagnosis of PTSD, but also yields a continuous score of frequency and severity for each symptom ranging from 0 to 188 (Blake, Weathers \& Nagy, 1995). The CAPS is able to assess both lifetime and current levels of PTSD while distinguishing between specific trauma and earlier lifetime traumas. The CAPS has been found to have a sensitivity of 0.81 and a specificity of 0.95 (Newman, Kaloupek, $\&$ Keane, 1996). Inter-rater reliability for interviewers in the larger study was assessed for the CAPS based on eight audio taped interviews, rated by a minimum of three interviewers. The intra-class correlation coefficient (ICC; Shrout \& Fliess, 1979) for the total PTSD score was 0.96 (Shea et al., 2010)

The LIFE is a semi-structured interview rating system for assessing the longitudinal course of axis-I mental disorders. The LIFE is divided into three sections: psychopathology, treatment history, and psychosocial functioning. It can be 
adapted to cover different time intervals and has frequently been used to assess six month or one year intervals. At the initial assessment the psychopathology section of the LIFE was administered to track the course of PTSD over the time period beginning with deployment to the present. The LIFE-base, which is a baseline version of the LIFE that assesses psychosocial functioning (current, best and worst over prior six months), was also administered at the initial assessment. The LIFE interview gathers detailed information regarding the presence and change in individual symptoms over the interval being assessed. This detailed information is recorded in the form of Psychiatric Status Ratings (PSRs) for each disorder present. Weekly ratings or PSRs are recorded for the interval covered using either a 6-point or 3-point scale. This scale indicates whether the individual meets full criteria, is in partial remission or in full remission from the given disorder at any point (Keller et al., 1987). The current study will use the 6-point scale for PTSD and 3-point scale for alcohol use disorders. The 6point scale for PTSD assesses both diagnostic criteria as well as the level of functional impairment and are distinguished as follows: $6=$ meets DSM-IV criteria for definite PTSD and the symptoms cause extreme impairment in functioning, $5=$ meets DSMIV criteria for definite PTSD, 4 = meets two of the three B, C, or D criteria and has clinically significant symptoms of impairment or distress from this disorder, $3=$ meets one of the three B, C, or D criteria with no more than moderate impairment or distress, $2=$ does not meet criteria $\mathrm{B}, \mathrm{C}$, and D but has one or more symptoms of the disorder in no more than a mild degree with no impairment or distress, and $1=$ none of the above. Previous studies have found that subjects accurately recall their clinical status over at minimum a six month interval (Warshaw \& Keller, 1994). 


\section{AUDs}

AUDs were assessed using the LIFE as explained above. The reason a 3-point scale is being used for AUDs compared to the 6-point PTSD scale is that the original study (Shea et al. 2010) focused much more detail on PTSD symptom severity and variability between individuals than it did with AUD. The 3-point PSRs for the AUDs are as follows: $1=$ symptom not present, $2=$ symptom is met at a sub-threshold level and $3=$ symptom is met at a full threshold level for that symptom. In order for the symptom to count towards the diagnosis, the symptom has to be at the "3" level. For dependence, respondents must report at least three symptoms at the "3" level and for abuse 1 symptom at the "3" level must be reported. PSR levels for dependence and abuse are then coded as " 3 " meets criteria for the disorder, "2" sub-threshold, "1" present in past but not present currently, and " 0 " never met full criteria for the disorder. Specific scoring criteria for AUD will be detailed separately for Aims 1 and 2 in the Proposed Analyses section.

\section{Combat Exposure}

Individual combat exposure was assessed by using the Hoge Combat Experiences Survey (HOGE). This self-report measure was designed to assess exposure to combat and other war zone experiences in Iraq and Afghanistan (Hoge et al., 2004). This five item measure assesses perceived threat (0-3), number of engagements with the enemy 0-999), personal injury (0-10) and other combat related exposures (0-52). The five items can be combined to give a continuous measure of one's perceived level of combat. The HOGE was administered at the initial 
assessment and at follow-up sessions to establish rater consistency. For the current study, only the baseline assessment will be used. 


\section{CHAPTER 4}

\section{FINDINGS}

\section{Preliminary Analysis}

Prior to examination of the study's substantive research questions, initial analyses examined the distributional properties of the primary measures (AUD, PTSD and CE) for skewness and kurtosis and to identify any patterns of missing data. The primary measures were all within acceptable ranges (e.g., Skewness > 2.0, Kurtosis > 4.0) and thus data transformation was not needed. In order to assess the possible effects of attrition on our data we used baseline rates of AUD, PTSD and combat exposure to compare the sample at $\mathrm{T} 1$ and $\mathrm{T} 3$ and found no evidence of differential attrition, $\mathrm{t}(236)=0.56<.58 ; \mathrm{t}(236)=.19<.85 ; \mathrm{t}(236)=1.89<.06 ;$ respectively While combat exposure approached levels of significance the mean level of combat exposure was lower in the attrition group than the group that remained through T3 (M $=7.98$ vs. $M=10.75$ ) suggesting that higher levels of combat exposure were not associated with attrition.

To facilitate tests of study hypotheses, summary variables were created. For tests of Aim 1, combat exposure was categorized using tertile splits to create combat exposure groups of low (CE1), moderate (CE2) and high (CE3) levels of reported combat exposure. The Longitudinal Interval Follow-up Evaluation's weekly psychiatric status ratings for both PTSD and AUDs were averaged across respective time points to produce values for T2 and T3. In contrast, the Clinician Administered 
PTSD Scale (CAPS) was a continuous variable produced from administered interviews at T1, T2, and T3. Additionally, AUD variables consisted of alcohol abuse, alcohol dependence and a combined value reflecting individuals with either abuse or dependence. Relationship status (RS) was coded as either single/divorced/widowed (RS1) or married/cohabitating (RS0).

Descriptive statistics for the sample including means and standard deviations for AUD, PTSD and combat exposure variables across time can be found in Table 1. To reiterate, the combat exposure assessment was only captured at $\mathrm{T} 1$ and those that dropped from the study actually reflected lower levels of exposure than those who remained throughout.

\section{Aim 1: Prospective Examination of PTSD and AUD}

To test Hypotheses 1 through 3, we conducted 2 X 3 X 3 (RS X CE X Time) analyses of variance (ANOVAs) on our primary measures of interest, the PTSD and alcohol use disorder variables. In support of Hypothesis 1a, combat exposure was significantly associated with PTSD, $F(2,158)=14.55, p<.001$. As expected, individuals with higher levels of combat exposure reported higher levels of PTSD symptomatology, see Figure $1(\mathrm{CE} 3: \mathrm{M}=28.91, \mathrm{SD}=21.61 ; \mathrm{CE} 2: \mathrm{M}=16.12, \mathrm{SD}=$ 17.82; $\mathrm{CE} 1: \mathrm{M}=12.07, \mathrm{SD}=12.68)$. Tukey's HSD test showed that participants in the high combat exposure group reported significantly higher levels of PTSD then the moderate and low level combat exposure groups $(\mathrm{p}<.05)$. In contrast to Hypothesis 1b, combat exposure was not associated with AUD symptoms. Likewise, in contrast to Hypotheses 2 and 3, relationship status did not moderate relations between combat exposure and either PTSD or AUD. We also observed a significant time effect on 
PTSD, $F(2,316)=11.71, p<.001$, with a significant decrease in symptoms over 12 months. Contrast effect analyses indicated significant decreases from T2 to T3, $F$ $(1,158)=6.40, p<.05$ and from T1 to T3, $F(1,158)=16.52, p<.001$ (Figure 2$)$.

Neither relationship status nor combat exposure interacted with time in the prediction of PTSD or AUD symptoms.

\section{Aim 2: Prospective Examination of Associations between PTSD and AUD}

\section{Symptomatology}

To examine hypothesized reciprocal associations between PTSD and AUD

(Hypothesis 4) we conducted cross-lagged panel path models with RS, CE and RS X

$\mathrm{CE}$ interaction as manifest exogenous variables. For these analyses, RS and CE were coded as described earlier. In separate models we examined alcohol abuse or dependence vs. dependence alone. Covariances between PTSD and AUD at Time 1 were estimated to control for cross-sectional associations between these factors and lag one autoregressive paths were estimated between like constructs over time (e.g., AUD T1 to AUD T2, PTSD T2 to PTSD T3). Endogenous manifest variables (Time 1

- Time 3) were also covaried. Controlling for both autoregressive paths and crosssectional associations allowed for a more statistically conservative estimation of prospective associations between endogenous variables (e.g., AUD T1 to PTSD T2, PTSD T2 to AUD T3). 
While we examined several parallel models substituting abuse, dependence or either abuse or dependence for the AUD variable, the following results depict the dependence variable which we elected to focus on due to its greater severity.

Similarly, PTSD was measured using both the LIFE and CAPS assessments and analyzed separately for both. Here we present results using the more thorough interview based CAPS assessment.

Our cross-lagged panel path analysis model demonstrated a good fit to the data, as indicated by a small and non-significant Chi-square value $\left(\chi^{2}[16, \mathrm{~N}=159]=\right.$ 9.72, $p=0.88)$, small residuals $(\mathrm{RMSEA}<.001)$, and large Fit Index values $(\mathrm{CFI}=$ 1.0, NNFI $=0.99)$. We observed a significant association between Time 1 AUD and Time 1 PTSD symptoms $(r=.21, p<.01)$ such that higher levels of PTSD symptoms were associated with higher levels of AUD symptoms. As can be seen in Figure 3, combat exposure was significantly associated with Time 1 PTSD symptoms $(\beta=.35$, $p<.01)$ such that higher levels of exposure resulted in higher levels of PTSD symptoms. Additionally, RS was significantly associated with Time 1 alcohol dependence $(\beta=.42, p<.05)$ such that individuals in a relationship were less likely to exhibit alcohol dependence than individuals who were single. No significant RS X CE interaction effects were observed. As can also be seen in Figure 3, we observed very strong autoregressive effects (e.g., AUD T1 to AUD T2, PTSD T2 to PTSD T3) 
ranging from .83 to .98 (all $p$ 's $<.001)$. After controlling for cross-sectional

associations between baseline PTSD and AUD symptoms and robust autoregressive effects, we observed no significant reciprocal relations between PTSD and AUD symptoms. Therefore, Hypothesis 4 was not supported.

In light of the very high autoregressivity in the three-wave model we examined a two wave model (T1-T3) to see if a longer measurement interval would facilitate prospective prediction by decreasing the autoregressive associations between PTSD and AUD across time. Similarly, this model demonstrated a good fit to the data, as indicated by a small and non-significant Chi-square value $\left(\chi^{2}[6, \mathrm{~N}=159]=3.18, p=\right.$ 0.77), small residuals $($ RMSEA $<.001)$, and large Fit Index values $(\mathrm{CFI}=1.0, \mathrm{NNFI}=$ 0.99). By increasing the time interval the autoregressive effects were reduced but remained very high (i.e., .84 for alcohol dependence, .74 for PTSD) and consistent with the three wave model the cross-lagged associations were still not significant.

\section{Aim 3: Influence of Combat Exposure on AUD and PTSD}

A cross lagged panel design was also used to analyze the prospective influence of combat exposure on AUD and PTSD symptomatology at Times 2 and 3. By controlling for cross-sectional associations at Time 1 (AUD, PTSD and CE), this model allowed for a cross lagged examination of combat exposure on both AUD and PTSD across time (e.g., CE to AUD T2, CE to PTSD T3). This model demonstrated a good fit to the data, as indicated by a small and non-significant $\chi^{2}(12, \mathrm{~N}=159)=5.77$, 
$p=0.93$, small residuals $(\mathrm{RMSEA}<.001)$, and large Fit Index values $(\mathrm{CFI}=1.0$, NNFI $=0.99$ ). In partial support of Hypothesis 5, after controlling for cross-sectional associations and strong autoregressive effects, combat exposure was significantly associated with PTSD at Time $3(\beta=.08, p<.05$, one-tailed $)$, such that higher levels of combat exposure were associated with higher levels of PTSD symptomatology at Time 3. 


\section{CHAPTER 5}

\section{CONCLUSION}

This study sought to build on prior research by prospectively examining relations between post-traumatic stress disorder and alcohol use disorder symptomatology in a sample of returning OIF/OEF deployed National Guard troops.

Overall support for our hypotheses was mixed. In support of Hypothesis 1a, combat exposure was significantly associated with PTSD but was not associated with AUD (Hypothesis 1b). For PTSD symptoms, we observed a significant Time effect, with decreases in reported symptomatology over the 12-month follow-up period. In contrast to Hypotheses 2 and 3, relationship status did not moderate relations between combat exposure with either PTSD or AUD. Cross-lagged panel path models indicated that combat exposure was significantly associated with Time 1 PTSD symptoms and that relationship status was significantly associated with Time 1 alcohol dependence. However, in contrast to Hypothesis 4, after controlling for significant cross-sectional associations between baseline PTSD and AUD symptoms and robust autoregressive effects, we observed no significant reciprocal prospective relations between PTSD and AUD symptoms. In partial support of Hypothesis 5, combat exposure was prospectively associated with PTSD symptoms at Time 3, indicating that over and above observed cross-sectional associations, greater combat exposure was associated with higher continuing levels of PTSD symptoms.

\section{Elaboration and Integration of Findings}


In review of the sample we found that it was comparable to samples used in other research studies in regards to PTSD, AUD and levels of combat exposure. According to the CAPS measure $11 \%$ of the sample met criteria for PTSD at Time 1 which included two of the eight female subjects. Based on the analyses used we did not focus specifically on cases of new onset after Time 1 but instead the overall trends in PTSD symptomatology. The LIFE measure reflected that at some point throughout the study that $24 \%$ of the sample met criteria for alcohol abuse and $22 \%$ met criteria for alcohol dependence. As discussed earlier the mean CE score for the sample was 10.05 with a range from 0-42 (out of 52). Based on the tertile splits this mean score reflects the moderate combat exposure group. Unfortunately it is hard to compare the use of this measure to other studies because previous research has not focused on this item of the HOGE assessment. Others studies have used the first item of the assessment which merely asks the number of firefights one has engaged in (Hoge et al., 2004) or used the number of deployments a service member had participated in. Therefore, it would be difficult to say whether this particular sample reported more or less combat exposures than previous studies.

Prior research has shown that military deployments and combat experiences specifically are a risk factor for the prevalence of PTSD (Hoge et al., 2004; Litz, 2007). Hoge and colleagues conducted a cohort study of post deployment mental health disorder prevalence compared to pre-deployment using different representative samples (Army \& Marine Infantry Brigades) and found that recently redeployed service members reported significantly higher prevalence of mental health disorders including depression, generalized anxiety and PTSD (27.9\% vs. $20.9 \%)$ than the non- 
deployed comparison groups with PTSD being the most significant (18.0\% vs. 9.4\%). A key factor identified using the Hoge questionnaire was the number of reported fire fights unit members had reported being engaged in which was representative of their individual combat exposure. The samples sizes for the study were 2,530 before deployment and 894 post-deployment using standardized mental health screens and self-report questionnaires (administered 3-4 months after deployment) (Hoge et al., 2004).

As noted, our findings replicate these effects, providing further evidence that the level of combat exposure plays an important predictive role in determining PTSD. While our study did not have a pre and post deployment comparison, we were able to use the same sample and clinician administered measures to produce more thorough and prospective findings.

However, unlike existing literature on alcohol use among returning veterans (Jacobson et al., 2008; Thomas et al., 2010; Wilk et al, 2010), we found no association between combat exposure and alcohol use disorders. In both the Thomas and Wilk studies, samples were collected from large anonymous surveys using alcohol screens to determine level of alcohol misuse and combat exposure. These studies found that higher levels of threatening situations were associated with alcohol misuse (Wilk et al., 2010) and that National Guard members reflected higher rates of alcohol misuse across time (3-12 months) compared to active duty members (Thomas et al., 2010). The Jacobson study was the only study that actually looked at pre-deployment and post-deployment comparisons using online questionnaires to explore associations between combat exposure and alcohol related behavior. They used a large sample of 
77,047 service members at baseline and compared follow-up assessments which included 37,310 non-deployed, 5,661 deployed without combat exposure and 5,510 with combat exposure. This multiservice sample answered standardized questionnaires to determine that Reserve and National Guard service members who deployed and reported combat exposures were significantly more likely to experience new-onset heavy drinking, binge drinking and alcohol related problems compared to non-deployed personnel (Jacobson et al., 2008).

There are many reasons why our study, in comparison to the existing literature, may have not found the same relations between combat exposure and alcohol use disorders. These factors include anonymous reporting, sample size and measures used. Our study used a much smaller sample and clinician administered measures to assess alcohol use disorders among participants leading to more conservative levels of analysis. The other factor is the importance of anonymity in reporting symptoms which has been shown to greatly impact the accuracy of prevalence and treatment of service members returning from deployment. Our study was confidential but previous studies have shown that service members are less honest in face to face reporting than by anonymous screens or surveys (Warner et al., 2011).

Despite previous research demonstrating the increasing dual diagnoses of AUDs and PTSD (Allison-Aipa et al., 2010; Jacobson et al., 2010; Thomas et al., 2010), beyond the replication of cross-sectional associations, our findings failed to yield new insights in to the dynamic nature of PSTD and AUD relations over time. Based on observations in the clinical literature that individuals with PTSD may abuse alcohol to self-medicate, we sought to extend prior cross-sectional research on 
relations between PTSD and AUD by examining these constructs prospectively. While definitive explanations of null effects are not possible, our ability to detect prospective effects was largely undermined by the very strong autoregressive effects we observed. These findings suggest the likely need for both longer measurement intervals and larger sample sizes to better elucidate these conceptually driven hypothesized reciprocal associations. As noted, we observed a significant time effect on PTSD, with decreases in endorsed symptoms over the 12-month follow-up period. In contrast, our mean scores for AUDs did increase over time but not in a significant manner in contrast to some previous studies (Jacobson et al., 2008; Thomas et al., 2010). As discussed next, in addition to the measurement interval issue noted above, there are numerous conceptual and methodological factors to consider with respect to our findings.

\section{Strengths and Limitations}

The current study possesses a number of substantial strengths. Chief among these is the study's prospective design with three assessments over a 12 month period. An additional important strength of this current study is the relevance of this sample given that assessments were conducted fairly soon after redeployment to capture the importance of the post-deployment transition period. Additionally, the inclusion of multiple Reserve and National Guard units allowed for the level of combat exposure to vary providing a varying sample of deployment experiences to help generalize our findings instead of it being limited to a particular type of unit or deployment location. Moreover, the assessment of PTSD and AUD using validated interviews such as the Clinician Administered PTSD Scale and the frequency of the Longitudinal Interval 
Follow-up Evaluation for both PTSD and AUDs also constitute substantial strengths. Therefore, this is one of the first studies to prospectively examine both PTSD and alcohol use disorders among recent redeployed veterans utilizing diagnostic interviews.

In contrast to these strengths, there are a number of limitations in the current study. There was substantial attrition (33\%) by Wave 3 of the study, but, importantly, no evidence of higher levels of PTSD or AUD symptoms among those who were lost to follow-up. While the relatively timely redeployment assessment was a strength of the study, there was some variability between when individuals received their first assessments. So while all participants had a baseline assessment at Time 1 with follow-up assessments at six months and 12 months, the range from when participants began their baseline was from within a month of redeployment to more than six months after redeployment, which could have easily masked some trends in the development of either AUD or PTSD.

Similarly, while prospering from a variety of units who had deployed and an adequate number of study participants there were certain demographic limitations that would prevent any gender or minority generalizations. While the military and the National Guard and Reserves of New England consist primarily of white males, our study sample is even less than representative of those organizations. Therefore, important questions about whether the prevalence of PTSD and AUD vary by race/ethnicity and gender, and whether these factors moderate the course of PTSD and AUD could not be addressed in the current study. An additional limitation for this case was the particular statistical analysis used did not make adjustments or estimates 
for missing data. So despite the evidence suggesting that data are missing at random, because of case-wise deletion of missing data the initial sample of 238 subjects at Time 1 was reduced to 159 when all waves were incorporated. Missing data estimation using recommended approaches such as maximum likelihood or multiple imputation estimations (Schafer \& Graham, 2002) would yield more powerful tests of study hypotheses. Additionally, the difficulty of detecting interaction and moderator effects in non-experimental research has been long recognized (McClelland \& Judd, 1993) and we were likely quite underpowered to detect the moderated effects investigated under Hypotheses 3.

A host of potential conceptual and methodological factors may have influenced results of this research. Conceptually, although widely recognized as co-morbid, several possible states of nature exist with respect to the co-occurrence of AUD and PTSD diagnoses and symptomatology. These include: (a) AUD secondary to PTSD; (b) PTSD secondary to AUD, (c) common diathesis; (d) bidirectional influence; and (e) overlapping diagnostic criteria (Wood, Vinson, \& Sher, 2001). In the current research we sought to better explicate questions related to order of onset and bidirectional influences but, as noted, our results are not informative with respect to these questions. Designs better suited to address these and related issues are discussed in the next section. Methodologically, in addition to the sample size and length of measurement interval limitations already noted, a number of other factors warrant consideration. These include concerns about the validity of self-report. Related to concerns about self-report, additional potential limitations relate to the reliability and 
validity of the assessment measures used here, the settings in which they were administered, and their consequent fidelity.

\section{Implications, Future Directions and Conclusions}

Given the recent proliferation of combat deployments that is unprecedented in the history of an all volunteer military, there is increasing interest and burgeoning research on the correlates and putative determinants of PTSD. Nonetheless, there is still very limited prospective examination of the long term development of both AUD and PTSD within this current military veteran population. Additional assurances of confidentiality for participants (e.g., Certificate of Confidentiality, more explicit assurances that reporting will not compromise performance evaluations) would also yield potential benefits for future research. The current study replicated prior crosssectional research on the effects of combat exposure on AUD symptoms and extended them to prospective prediction of combat exposure on AUD symptoms at 12 months. We also observed interesting findings related to relationship status, alcohol dependence associations and the significant decrease of PTSD symptoms over time. Future research with large and more diverse samples that includes pre-deployment assessment of AUD, alcohol involvement, PTSD, and other risk factors along with multiple follow-ups with longer measurement intervals are needed to build on this and other prior research. 


\section{Tables}

Table 1

Descriptive statistics for AUD, PTSD and Combat Exposure

\begin{tabular}{|c|c|c|c|c|c|c|}
\hline \multirow[b]{2}{*}{ Variable } & \multicolumn{2}{|c|}{$\underline{\text { Time } 1}$} & \multicolumn{2}{|c|}{ Time 2} & \multicolumn{2}{|c|}{ Time 3} \\
\hline & $\underline{\mathrm{M}}$ & $\underline{\mathrm{SD}}$ & $\underline{\mathrm{M}}$ & $\underline{\mathrm{SD}}$ & $\underline{\mathrm{M}}$ & $\underline{\mathrm{SD}}$ \\
\hline AUD & 0.58 & 0.95 & 0.65 & $\overline{0.95}$ & 0.67 & 0.94 \\
\hline PTSD & 22.51 & 20.21 & 20.11 & 20.16 & 17.65 & 19.75 \\
\hline $\mathrm{CE}$ & 10.05 & 9.54 & & & & \\
\hline
\end{tabular}

Note. AUD $=$ PSR rating (0-3) for either alcohol abuse or dependence; PTSD = CAPS score (0-188); $\mathrm{CE}=$ HOGE Item 5 score $(0-52)$ 


\section{Figures}

Figure 1: CE effect on PTSD

\section{CE on PTSD}

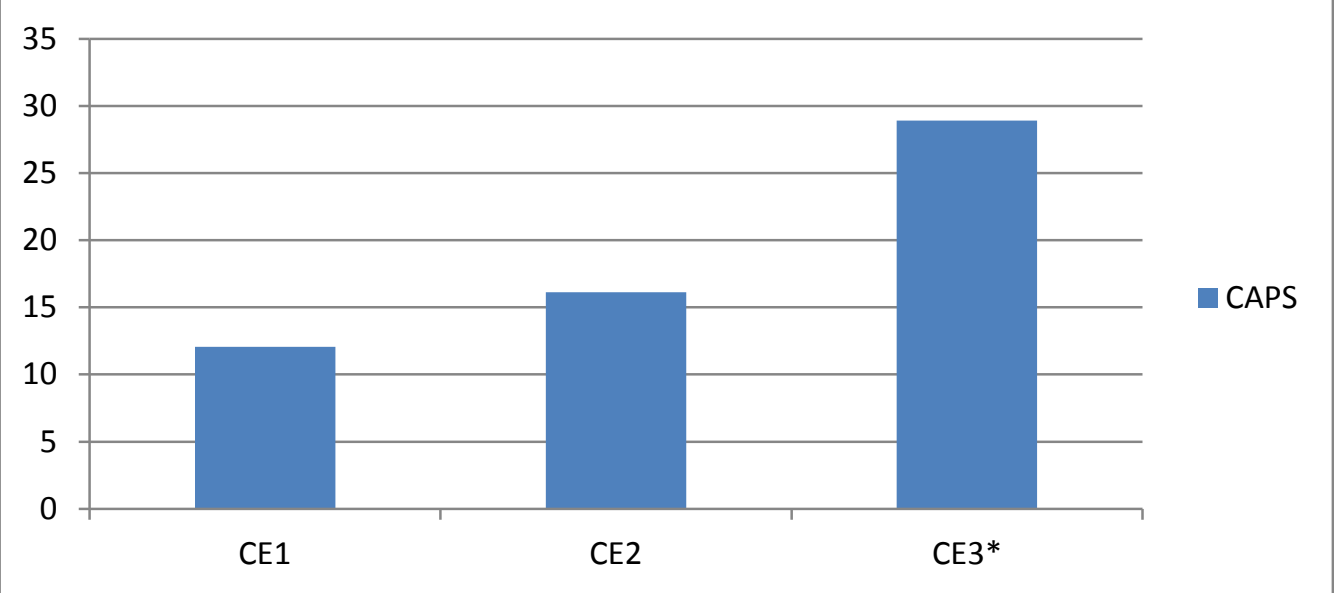

* High combat exposure group (CE3) reported significantly higher levels of PTSD then the moderate (CE2) and low level (CE1) combat exposure groups ( $<$.05).

Figure 2: Time effect on PTSD

\section{Time on PTSD}

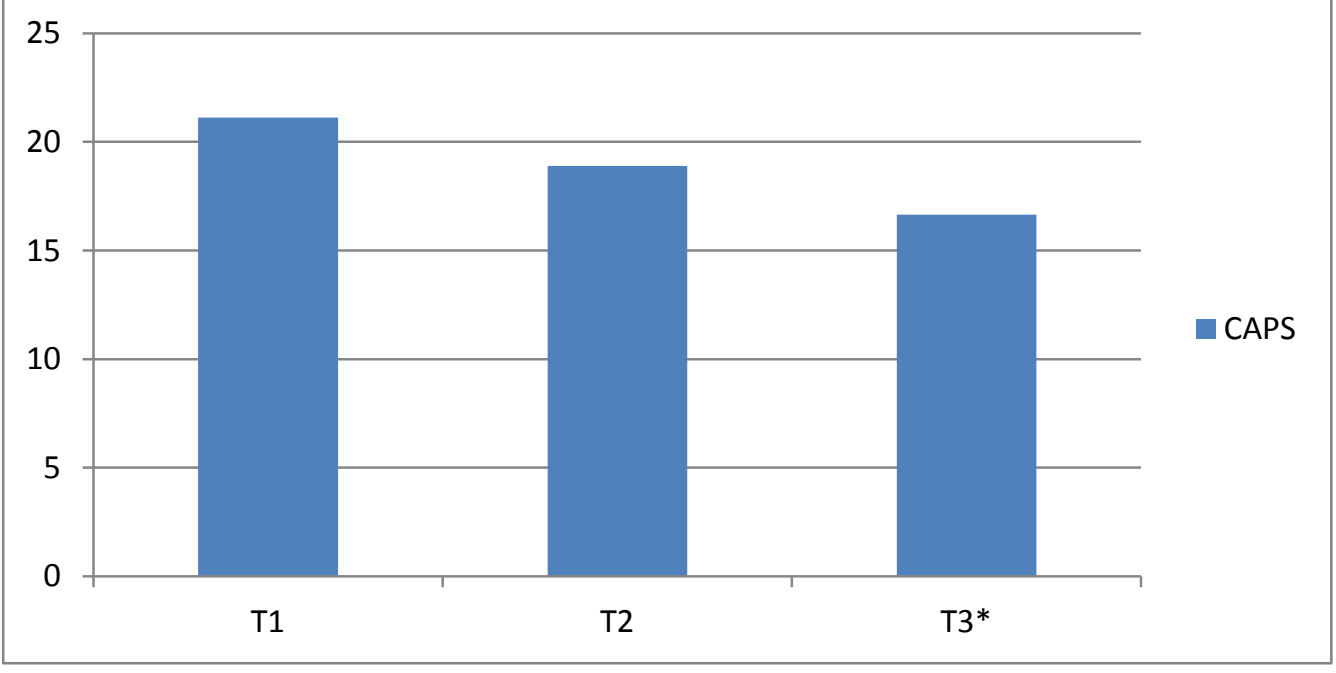

*Contrast effect analyses indicated significant decreases from T2 to T3, $F(1,158)=6.40, p<$ .05 and $\mathrm{T} 1$ to $\mathrm{T} 3, F(1,158)=16.52, p<.001$. 
Figure 3: Cross-lagged Panel Path Analysis Model

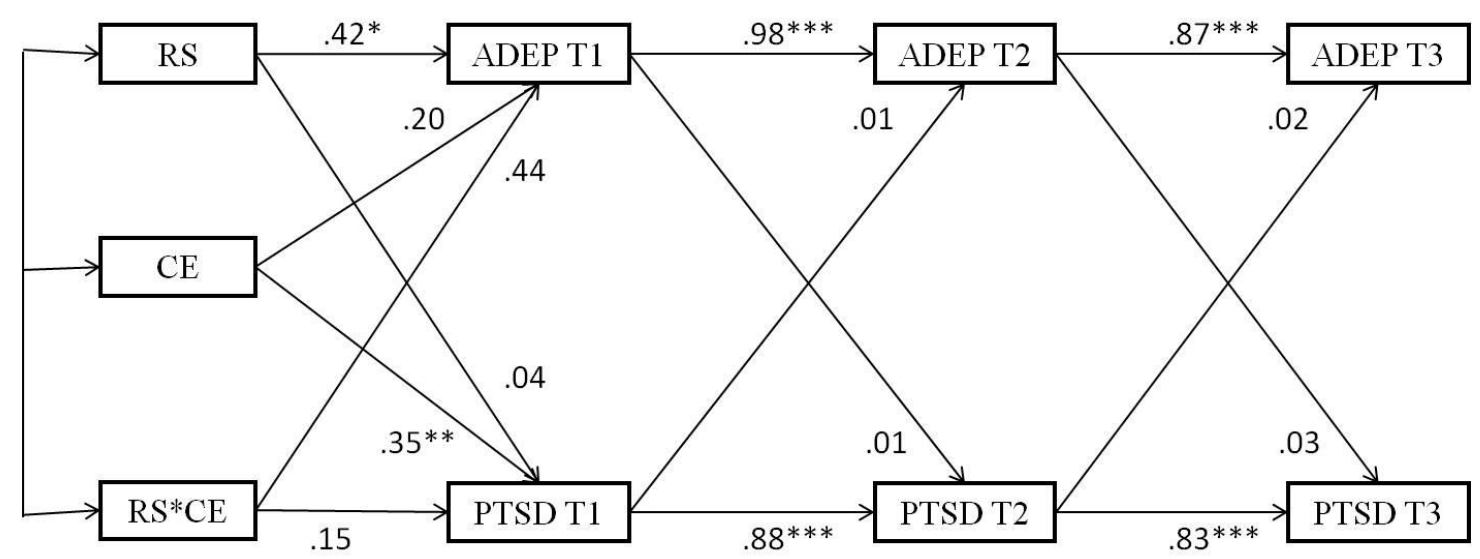

$* \mathrm{p}<.05, * * \mathrm{p}<.01, * * * \mathrm{p}<.001$ 


\section{BIBLIOGRAPHY}

Allison-Aipa, T.S., Ritter, C., Sikes P., \& Ball S. (2010). The impact of deployment on the psychological health status, level of alcohol consumption, and use of psychological health resources of post-deployed U.S. Army reserve soldiers. Journal of Military Medicine, 175(9), 630-637.

American Psychiatric Association: Diagnostic and Statistical Manual of Mental Disorders, Fourth Edition, Text Revision. Washington DC, American Psychiatric Association, 2000.

American Psychiatric Association: Diagnostic and Statistical Manual of Mental Disorders, Third Edition. Washington DC, American Psychiatric Association, 1980.

Andrews, B., Brewin, C.R., Stewart, L., Philpott, R., \& Hejdenberg, J. (2009). Comparison of immediate-onset and delayed-onset posttraumatic stress disorder in military veterans. Journal of Abnormal Psychology, 118(4), 767-777. doi:10.1037/a0017203

Blake, D.D., Weathers, F.W., Nagy, L.M., \& Kaloupek, D.G. (1995). The development of a clinician-administered PTSD scale. Journal of Traumatic Stress, 8, 75-90. doi:10.1002/jts.2490080106

Bliese, P.D., Wright, K.M., Adler, A.B., Thomas, J.L., \& Hoge, C.W. (2007). Timing of postcombat mental health assessments. Psychological Services, 4(3), 141-148. doi:10.1037/1541-1559.4.3.141

Bray, R. M., Marsden, M.E., \& Peterson, M.R. (1991). Standardized comparisons of the use of alcohol, drugs, and cigarettes among military personnel and civilians. American Journal of Public Health, 81, 865-869.

Bray, R.M., Pemberton, M.R., Lane, M.E., Hourani, L.L., Mattiko, M.J., \& Babeu, L.A. (2010). Substance use and mental health trends among U.S. Military active duty personnel: Key findings from the $2008 \mathrm{DoD}$ health behavior survey. Military Medicine, 175, 6:390.

Browne, T., Hull, L., Horn, O., Jones, M., Murphy, D., Fear, N.T., ... Hotopf, M. (2007). Explanations for the increase in mental health problems in UK reserve forces who have served in Iraq. British Journal of Psychiatry, 190, 484-489. doi:10.1192/bjp.bp.106.030544

Calhoun, P.S., Elter, J.R., Jones, E.R, Kudler, H., \& Straits-Troster, K. (2008). Hazardous alcohol use and receipt of risk-reduction counseling among U.S. veterans of the wars in Iraq and Afghanistan. Journal of Clinical Psychiatry, 69(11), 1689-1693. 
Fear, N.T., Iversen, A., Meltzer, H., Workman, L., Hull, L., Greenberg, N., ... Wessely, S. (2007). Patterns of drinking in the UK Armed Forces. Society for the Study of Addiction, 102, 1749-1759.

Fontana, A., \& Rosenheck, R. (2010). War zone veterans returning to treatment: Effects of social functioning and psychopathology. Journal of Nervous \& Mental Disease, 198(10), 699-707. doi:10.1097/NMD.0b013e3181f4ac88

Grant, B.F., Dawson, A.D., Stinson, F.S., Chou, S.P., Dufour, M.C., \& Pickering, R.P. (2006). The 12-Month Prevalence and Trends in DSM-IV Alcohol Abuse and Dependence. Alcohol Research and Health, 29(2), 79-91.

Hawkins, E.J., Lapham, G.T., Kivlahan, D.R., \& Bradley, K.A. (2010). Recognition and management of alcohol misuse in OEF/OIF and other veterans in the VA: a cross-sectional study. Drug and Alcohol Dependence, 109(1-3), 147-153.

Hoge, C.W., Castro, C.A., Messer, S.C., McGurk, D., Cotting, D.I., \& Koffman, R.L. (2004). Combat duty in Iraq and Afghanistan, mental health problems, and barriers to care. The New England Journal of Medicine, 351(1), 13-22.

Jacobson, I.G., Ryan, M.A.K., Hooper, T.I., Smith, T.C., Amoroso, P.J., Boyko, E.J., ... Bell, N.S. (2008). Alcohol use and alcohol-related problems before and after military combat deployment. Journal of American Medical Association, 300(6), 663-673.

Kim, P.Y., Thomas, J.L., Wilk, J.E., Castro, C.A., \& Hoge, C.W. (2010). Stigma, barriers to care, and use of mental health services among active duty and National Guard soldiers after combat. Psychiatric Services, 61(6), 582-588.

Keller, M.B., Lavori, P.W., Friedman, B., Nielson, E., Endicott, J., McDonald-Scott, P., \& Andereason, N.C. (1987). The Longitudinal Interval Follow-Up Evaluation. Archives of General Psychiatry, 44, 540-548.

Kulka, R.A., Schlenger, W.A., Fairbanks, J.A., Hough, R.L., Jordan, B.K., Marmar, C.R., \& Cranston, A.S. (1990). Trauma and the Vietnam War generation: Report of findings from the National Vietnam Veterans Readjustment Study. New York: Brunner/Mazel.

Leeies, M., Pagura, J., Sareen, J., \& Bolton, J. M. (2010). The use of alcohol and drugs to self-medicate symptoms of posttraumatic stress disorder. Depression and Anxiety, 27(8), 731-736.

Litz, B.T. (2007). Research on the impact of military trauma: current status and future directions. Military Psychology, 19(3), 217-238.

McClelland, G.H. \& Judd, C.M. (1993). Statistical difficulties of detecting interactions and moderator effects. Psychological Bulletin, American Psychological Association, 114 (2), 376-390. 
McDevitt-Murphy, M.E., William, J.L., Bracken, K.L, Fields, J.A., Monahan, C.J., \& Murpy, J.G. (2010). PTSD symptoms, hazardous drinking, and health functioning among U.S. OEF and OIF veterans presenting to primary care. Journal of Traumatic Stress, 23(1), 108-111.

Milliken, C.S., Auchterlonie, J.L., \& Hoge, C.W. (2007). Longitudinal Assessment of Mental Health Problems Among Active and Reserve Component Soldiers Returning from the Iraq War. Journal of American Medical Association, 298(18), 2141-2148.

National Comorbidity Survey. (2005). NCS-R appendix tables: Table 1. Lifetime prevalence of DSM-IV/WMH-CIDI disorders by sex and cohort. Table 2. Twelvemonth prevalence of DSM-IV/WMH-CIDI disorders by sex and cohort. Accessed at: http://www.hcp.med.harvard.edu/ncs/publications.php.

National Institute of Alcohol Abuse and Alcoholism. (2004). NIAAA Council Approves Definition of Binge Drinking. Volume Publication No. 04-5346. NIAAA Newsletter 2004; 3: 3.

Newman, E., Kaloupek, G.G., \& Keane, T.M. (1996). Assessment of posttraumatic stress disorder in clinical and research settings. In B.A. Van der Kolk ACM and L Weisaeth (Eds), Traumatic Stress: the effect of overwhelming experience on mind, body and society (pp. 242-275). New York, NY: Guilford Press.

Schafer, J. L., \& Graham, J. W. (2002). Missing data: Our view of state of the art. Psychological Methods, 7, 147 - 177.

Seal, K. H., Bertenthal, D., Miner, C. R., Sen, S., \& Marmar, C. (2007). Bringing the war back home: Mental health disorders among 103,788 US Veterans returning from Iraq and Afghanistan seen at Department of Veterans Affairs facilities. Archives Internal Medicine, 167(5), 476-482.

Shea, M.T., Vujonovic, A.A., Mansfield, A.K., Sevin, E., \& Liu, F., (2010). Posttraumatic stress disorder symptoms and functional impairment among OEF and OIF National Guard and reserve veterans. Journal of Traumatic Stress, 23(1), 100-107.

Shephard, Ben (2001). A war of nerves: soldiers and psychiatrists in the twentieth century. Cambridge, Massachusetts, Harvard University Press.

Shrout, T.C. \& Fleiss, J.L. (1979). Intraclass Correlations: Uses in assessing rater reliability. Psychology Bulletin, 86, 420-428.

Stahre, M.A., Brewer, R.D., Fonseca, V.P., \& Naimi, T.S. (2009). Binge drinking among U.S. active-duty military personnel. American Journal of Preventive Medicine, 36(3), 208-217. doi:10.1016/j.amepre.2008.10.017 
Thomas, J.L., Wilk, J.E., Riviere, L.A., McGurk, D., Castro, C.A., \& Hoge, C.W. (2010). Prevalence of mental health problems and functional impairment among active component and National Guard Soldiers 3 and 12 months following combat in Iraq. Archives of General Psychiatry, 67(6), 614-623.

Warner, C.H., Appenzeller, G.N., Grieger, T., Belenkiy, S., Breitbach, J., Parker, J., ... Hoge, C. (2011). Importance of anonymity to encourage honest reporting in mental health screening after combat deployment. Archives of General Psychiatry, 68(10), 1065-1071. doi:10.1001/archgenpsychiatry.2011.112

Warshaw, M.G., Keller, M.B., \& Stout, R.L. (1994). Reliability and validity of the Longitudinal Interval Follow-Up Evaluation for assessing outcome of anxiety disorders. Journal of Psychiatric Research, 28, 531-545.

Wilk, J., Bliese, P.D., Kim, P.Y., Thomas, J.L., McGurk, D., \& Hoge, C.W. (2010). Relationship of combat experiences to alcohol misuse among U.S. Soldiers returning from the Iraq war. Journal of Drug and Alcohol Dependence, 108(1-2), 115-121. doi:10.1016/j.drugalcdep.2009.12.003

Wood, M.D., Vinson, D.C., \& Sher, K.J. (2001). Alcohol use and misuse. In A. Baum, T.A. Revenson \& J.E. Singer (Eds.), Handbook of health psychology (pp. 280318). Mahwah, New Jersey: Lawrence Erlbaum Associates. 